\title{
Sub-Doppler Double-Resonance Spectroscopy of Methane Using a Frequency Comb Probe
}

\author{
Aleksandra Foltynowicz $\odot,{ }^{1, *}$ Lucile Rutkowski $\odot,{ }^{2}$ Isak Silander $\odot,{ }^{1}$ Alexandra C. Johansson, ${ }^{1}$ \\ Vinicius Silva de Oliveira ${ }^{1},{ }^{1}$ Ove Axner®e, ${ }^{1}$ Grzegorz Soboń® ${ }^{3}$ Tadeusz Martynkien, ${ }^{4}$ \\ Paweł Mergo, ${ }^{5}$ and Kevin K. Lehmann ${ }^{6, \dagger}$ \\ ${ }^{1}$ Department of Physics, Umeå University, 90187 Umeå, Sweden \\ ${ }^{2}$ Université de Rennes, CNRS, IPR (Institut de Physique de Rennes)-UMR 6251, F-35000 Rennes, France \\ ${ }^{3}$ Laser and Fiber Electronics Group, Faculty of Electronics, Wroctaw University of Science and Technology, 50-370 Wroclaw, Poland \\ ${ }^{4}$ Faculty of Fundamental Problems of Technology, Wrocław University of Science and Technology, 50-370 Wroclaw, Poland \\ ${ }^{5}$ Laboratory of Optical Fiber Technology, Maria Curie-Sklodowska University, 20-031 Lublin, Poland \\ ${ }^{6}$ Departments of Chemistry and Physics, University of Virginia, Charlottesville, Virginia 22904, USA
}

(Received 27 January 2020; revised 9 July 2020; accepted 3 December 2020; published 11 February 2021)

\begin{abstract}
We report the first measurement of sub-Doppler molecular response using a frequency comb by employing the comb as a probe in optical-optical double-resonance spectroscopy. We use a $3.3 \mu \mathrm{m}$ continuous wave pump and a $1.67 \mu \mathrm{m}$ comb probe to detect sub-Doppler transitions to the $2 \nu_{3}$ and $3 \nu_{3}$ bands of methane with $\sim 1.7 \mathrm{MHz}$ center frequency accuracy. These measurements provide the first verification of the accuracy of theoretical predictions from highly vibrationally excited states, needed to model the high-temperature spectra of exoplanets. Transition frequencies to the $3 \nu_{3}$ band show good agreement with the TheoReTS line list.
\end{abstract}

DOI: 10.1103/PhysRevLett.126.063001

Methane is the first organic molecule detected in the atmosphere of hot-Jupiter exoplanets via satellite- and ground-based observations [1,2]. The observed spectra carry information about the conditions in the exoplanet's atmosphere, photochemistry, and planetary formation. To extract this information, accurate theoretical models of the gas-phase high-temperature spectra are needed, verified by high-precision laboratory measurements [3,4]. Theoretical spectra can be produced using the parameters from the ExoMol $[5,6]$ or TheoReTS $[7,8]$ line lists obtained from $a b$ initio calculations, but firmly assigned experimental transitions from highly excited levels are needed to judge the accuracy of these predictions. Rotationally resolved spectra of methane have been reported up to $16180 \mathrm{~cm}^{-1}$ [9], but at present only those up to the lower part of the Icosad polyad $\left(6800 \mathrm{~cm}^{-1}\right)$ are satisfactorily assigned $[10,11]$. Reaching highly excited levels by thermal excitation requires heating the sample to high temperatures $(\sim 1000 \mathrm{~K})$, which leads to congested spectra that are difficult to assign. Recently, the TheoReTS line list was found to provide good agreement with high-temperature experimental spectra up to the Icosad range $\left(7600 \mathrm{~cm}^{-1}\right)$

Published by the American Physical Society under the terms of the Creative Commons Attribution 4.0 International license. Further distribution of this work must maintain attribution to the author(s) and the published article's title, journal citation, and DOI. Funded by Bibsam.
$[12,13]$ and with low- and room-temperature spectra recorded in the $9000 \mathrm{~cm}^{-1}$ region [14]. However, firmly assigned experimental hot band transitions reaching levels above $8000 \mathrm{~cm}^{-1}$ are needed to allow empirical corrections to line positions in the Triacontad range and thus more accurate modeling of the high-temperature spectra $[13,15]$.

Optical-optical double-resonance (DR) spectroscopy [16] is a powerful tool for assignment of highly excited energy levels [17]. It provides a way to use an already assigned transition to unambiguously identify the lower or upper state quantum numbers of measured spectra. In optical-optical DR spectroscopy a saturating pump laser transfers the population of a single quantum state into another state, and a weaker probe laser measures transitions from (to) the selectively populated (depopulated) states. Figure 1(a) shows the case when the pump addresses transitions in the fundamental $\mathrm{C}$ $\mathrm{H}$ stretching band and the probe frequency is roughly twice the pump frequency. Two types of DR excitations can be observed: V-type excitations, in which the pump and probe transitions share a common depleted rotational state in the ground vibrational states, and ladder-type excitations, in which the upper level of the pump transition is the lower level for the probe. When a monochromatic pump is used, only a narrow velocity group of molecules is excited, and the resulting probe transitions are free of Doppler broadening [18]. This is particularly advantageous for $\mathrm{CH}_{4}$, where many transitions overlap in the Doppler-broadened spectra. If the pump transition is already assigned, the lower state quantum number of the DR probe transitions is known. Moreover, the 
(a) cw probe

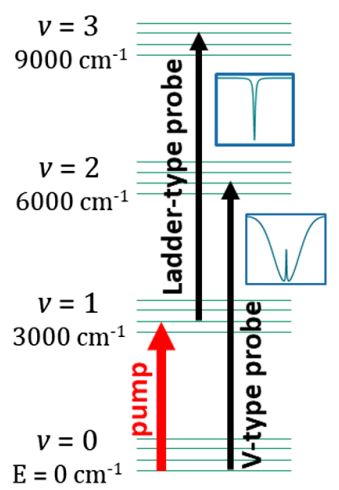

(b) comb probe

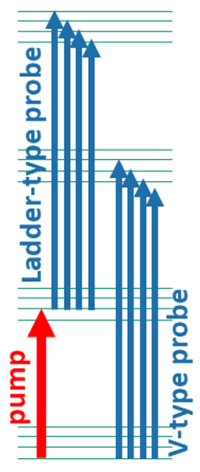

FIG. 1. The energy level structure of the $\nu_{3} \mathrm{C}-\mathrm{H}$ stretching bands of methane with pump (red) and probe transitions indicated for (a) a single frequency probe (black) and (b) a frequency comb probe (blue).

ratio of the probe transition intensities measured with different relative polarizations of the two fields depends upon the change in rotational quanta of the pump and probe transitions [19] and can be used to assign the quantum number of the upper state. In V-type excitations, the probe transitions appear in the linear absorption spectrum as subDoppler dips in the Doppler-broadened lines sharing lower level with the pump. The ladder-type probe transitions, on the other hand, appear in the spectrum as sub-Doppler absorption lines and allow one to reach levels that are not allowed from the ground vibrational state.

Optical-optical double-resonance spectroscopy of $\mathrm{CH}_{4}$ reaching the states in the $3 \nu_{3}$ band was previously reported by de Martino et al. [20-22] using nanosecond pump and probe pulses in the 1.6 and $3.3 \mu \mathrm{m}$ spectral regions, respectively. Both the sensitivity (few percent absorption noise) and the spectral resolution $\left(>0.12 \mathrm{~cm}^{-1}\right)$ were limited by the optical sources and not sufficient to accurately resolve the narrow transitions. Very recently, Okubo et al. [23] used $3.3 \mu \mathrm{m}$ continuous wave pump and probe lasers referenced to a frequency comb to determine frequencies of ten $Q$-branch transitions to the $2 \nu_{3}$ band with accuracy of a few tens of kilohertz. However, the spectral coverage of the probe was only $0.18 \mathrm{THz}\left(5.3 \mathrm{~cm}^{-1}\right)$, which makes searching for multiple unknown narrow probe transitions impractical.

Here we use a high-power $3.3 \mu \mathrm{m}$ continuous wave pump and a $1.67 \mu \mathrm{m}$ frequency comb probe [see Fig. 1(b)] to record sub-Doppler DR transitions of methane over $6 \mathrm{THz}\left(200 \mathrm{~cm}^{-1}\right)$ of bandwidth with $1.7 \mathrm{MHz}$ $\left(5.6 \times 10^{-5} \mathrm{~cm}^{-1}\right)$ center frequency accuracy. The V-type probe transitions allow precise determination of center frequencies of transitions to the $2 \nu_{3}$ band and we compare them to results of previous studies [24,25] to confirm the measurement accuracy. The ladder-type probe transitions reach rovibrational states in the $\sim 9000 \mathrm{~cm}^{-1}$ energy region and we compare their frequencies to predictions from the TheoReTS database [7].

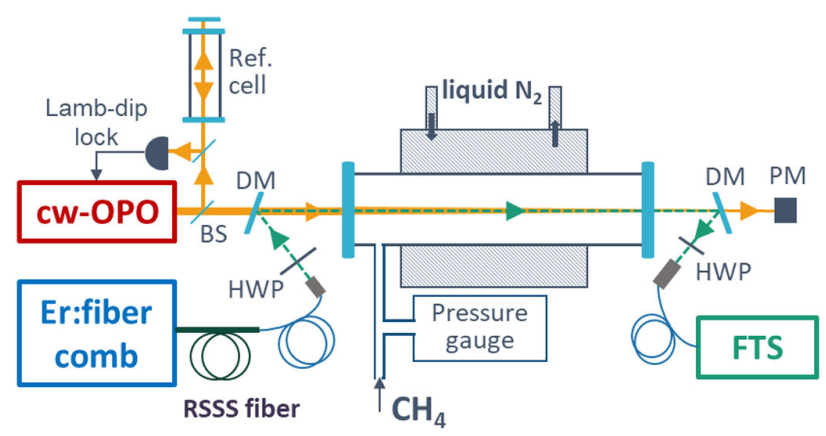

FIG. 2. Experimental setup. BS, beam splitter; DM, dichroic mirror; HWP, half-wave plate; RSSS fiber, Raman soliton selffrequency shift microstructured silica fiber; PM, power meter.

The experimental setup is shown in Fig. 2. The pump was a singly resonant continuous wave optical parametric oscillator (cw-OPO, Aculight, Argos 2400 SF, module C) with idler tunable in the 3.1-3.7 $\mu \mathrm{m}$ range with power up to $1 \mathrm{~W}$. The idler frequency was first tuned to the vicinity of the selected pump transition in the $\nu_{3}$ fundamental band using a wave meter (Burleigh, WA-1500-NIR-89, $\pm 0.0001 \mathrm{~nm}$ resolution, $\pm 2 \times 10^{-7}$ absolute relative accuracy) that monitored the pump and signal frequencies. Next, the idler frequency was stabilized to the center of the addressed transition using a frequency modulated Lambdip error signal from a reference cell filled with pure $\mathrm{CH}_{4}$ (see Ref. [26], for details of the stabilization scheme). This lock provided long-term frequency stability of the idler of $\pm 1 \mathrm{MHz}$, limited by a drift of the offset of the error signal. This translates into a maximum uncertainty of the probe transitions of $\pm 2 \mathrm{MHz}$, and we assume that the $1 \sigma$ uncertainty is equal to $2 / 3$ of that, i.e., $\pm 1.33 \mathrm{MHz}$. In total, DR spectra were recorded with the pump locked to nine different transitions in the $\nu_{3}$ fundamental band, viz. $R(0), R(1), P(1), Q(1), P(2, \mathrm{E}), P\left(2, F_{2}\right), P\left(3, A_{2}\right)$, $P\left(3, F_{1}\right)$, and $P\left(3, F_{2}\right)$.

The probe was an amplified Er:fiber frequency comb (Menlo Systems, FC1500-250-WG) with $250 \mathrm{MHz}$ repetition rate and $400 \mathrm{~mW}$ output power around $1.55 \mu \mathrm{m}$. The comb spectrum was shifted to cover $55 \mathrm{~nm}\left(200 \mathrm{~cm}^{-1}\right)$ around $1.67 \mu \mathrm{m}\left(6000 \mathrm{~cm}^{-1}\right)$ with $20 \mathrm{~mW}$ of power using a custom-made polarization-maintaining Raman soliton selffrequency shift microstructured silica fiber [27]. The comb was rf-stabilized to a GPS-referenced Rb oscillator, as described in Ref. [26]. The carrier envelope offset frequency was fixed at $20 \mathrm{MHz}$, while the repetition rate, $f_{\text {rep }}$, was locked to a tunable direct digital synthesiser that allowed stepping of the comb mode frequencies during spectral acquisition.

A sample of pure $\mathrm{CH}_{4}$ was contained in an 80-cm-long single-pass cell with $\mathrm{CaF}_{2}$ windows. The central $55-\mathrm{cm}-$ long part was liquid-nitrogen-cooled to increase the absorption signal. The pump and probe beams were combined using a dichroic mirror in front of the cell and separated using a similar mirror behind the cell. The OPO beam was 
collimated with a radius of $\sim 1 \mathrm{~mm}$ along the length of the cell, while the comb beam had a radius of $0.36 \mathrm{~mm}$ at the waist in the middle of the cell. The power of the transmitted pump beam was monitored using a power meter. The transmitted probe beam was coupled into a polarizationmaintaining fiber and led to a home-built fast-scanning Fourier transform spectrometer (FTS) with autobalanced detection [28]. A zero-order half-wave plate with design wavelength of $1550 \mathrm{~nm}$ was used in front of the cell to adjust the polarization of the probe to be parallel or perpendicular to the pump polarization. The second zero-order half-wave plate, positioned after the cell, was used to align the polarization of the comb light to the slow axis of the polarization-maintaining fiber.

The pressure in the cell was adjusted so that the transmission of the pump power on resonance was $20 \%$, which was found to yield a maximum V-type probe signal. This corresponded to 30-60 mTorr for the different pump transitions. All pump transitions were strongly saturated, with the on-axis intensity at the entrance of the cell 30-340 times above saturation. The experimental conditions for all pump transitions are summarized in Ref. [26].

The nominal resolution of the FTS was set to $250 \mathrm{MHz}$ to enable precise measurements of comb mode intensities without the influence of the instrumental line shape $[29,30]$. One interferogram was acquired in $5.8 \mathrm{~s}$ and yielded a sample point spacing of $250 \mathrm{MHz}$. The $f_{\text {rep }}$ was then tuned 125 times in steps of $2.78 \mathrm{~Hz}$, which resulted in a $2 \mathrm{MHz}$ shift of the comb modes per step. These 125 spectra were background corrected, interleaved, and averaged (up to 16 times) to yield a noise equivalent absorption coefficient of $7.2 \times 10^{-6} \mathrm{~cm}^{-1}$ after $3.2 \mathrm{~h}$ averaging. The measurement was then repeated with the probe polarization rotated by $90^{\circ}$.

Figure 3 shows the probe spectrum recorded with the pump locked to the $R(0)$ transition in the $\nu_{3}$ fundamental band and with perpendicular pump-probe polarizations. Most of the lines in the spectrum are Doppler-broadened transitions in the $2 \nu_{3}$ overtone band. A fit to the Dopplerbroadened spectrum, described in detail in Ref. [26], revealed that the sample temperature was 111(4) K, i.e., significantly above $77 \mathrm{~K}$. We attribute this to inefficient cooling of the cell and contributions from the warmer gas in the uncooled part of the cell (temperature retrieved from a spectrum with pump beam off was found to be $100 \mathrm{~K}$ ), as well as to the heating by the pump beam (predicted to yield $7 \mathrm{~K}$ temperature increase).

The inset in Fig. 3 shows an enlargement of the $2 \nu_{3}$ overtone $R(0)$ transition, in which the sub-Doppler V-type probe transition is visible. This transition has a full width at half maximum of 12.9 (2) $\mathrm{MHz}$ and a depth of $40 \%$ relative to the Doppler-broadened line. The predicted width and depth of this transition are $8 \mathrm{MHz}$ and 50\%, respectively, where the width is dominated by the power broadening of the pump transition. This indicates that the probe transition may be additionally broadened by the frequency jitter of the pump.

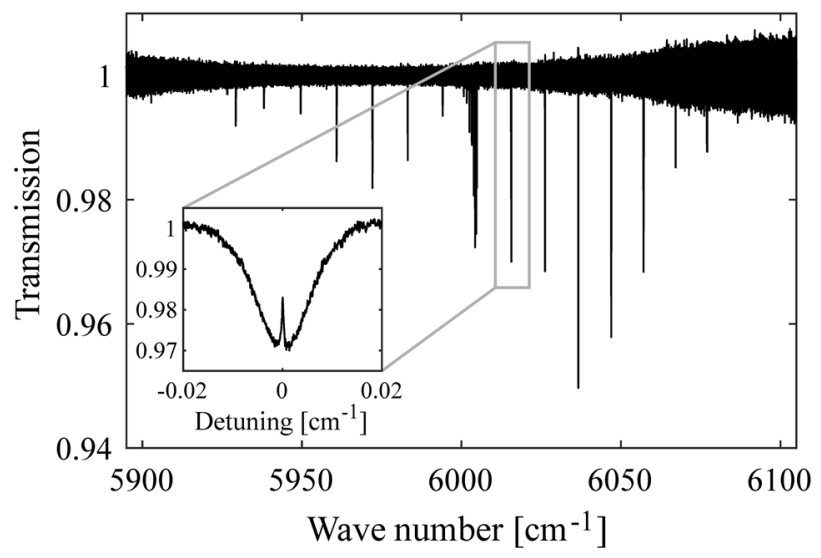

FIG. 3. The $2 \nu_{3}$ overtone and DR spectrum of 30 mTorr of methane measured with the comb probe when the pump is locked to the fundamental $R(0)$ transition and the relative pump-probe polarizations are perpendicular. The inset shows the V-type probe transition in the $2 \nu_{3}$ overtone $R(0)$ line.

In total, for the nine different pump transitions we detected V-type resonances in 18 different overtone transitions, listed in Table II in Ref. [26]. The center frequencies of five of these V-type resonances, obtained from a fit of a Lorentzian function, can be compared to the center frequencies of the corresponding $2 \nu_{3}$ overtone transitions measured in the Doppler-limited regime by Zolot et al. [25] using dual comb spectroscopy (DCS). This comparison is shown in Fig. 4, where the error bars are combinations of the uncertainties originating from the fit and the frequency stability of the pump, and the uncertainties reported for the DCS measurements, which are on the order of 0.2 $0.6 \mathrm{MHz}\left(0.6-2 \times 10^{-5} \mathrm{~cm}^{-1}\right)$. The agreement between the two measurements is very good, with the rms value of the discrepancies equal to $0.35 \mathrm{MHz}$, indicating that the frequency accuracy of the DR measurements is better than $1 \mathrm{MHz}$. The $R(0)$ and $Q(1)$ transition frequencies were

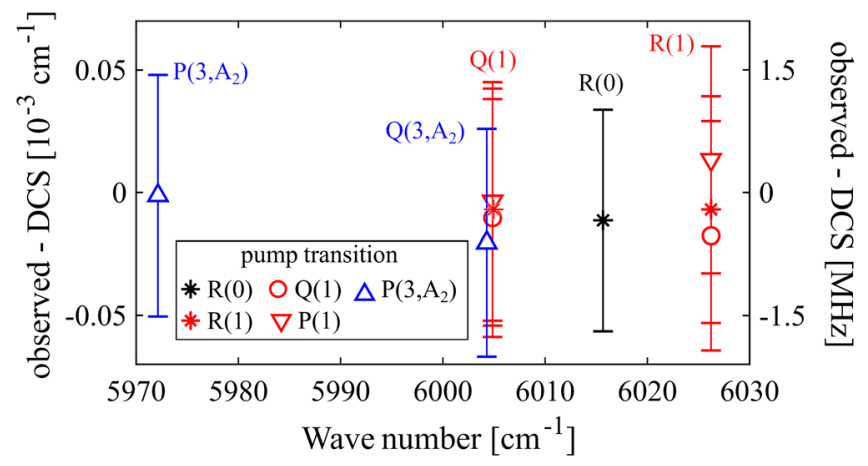

FIG. 4. The difference between frequencies of the observed sub-Doppler V-type probe transitions in the $R(0), R(1), Q(1)$, $Q\left(3, A_{2}\right)$, and $P\left(3, A_{2}\right)$ lines in the $2 \nu_{3}$ overtone band (detected with pump locked to five different fundamental transitions, as marked in the legend) and the $2 \nu_{3}$ overtone transitions from Ref. [25]. The left axis is in wave numbers, while the right axis is in megahertz. Error bar-see text. 
previously also measured with submegahertz accuracy using sub-Doppler spectroscopy [24], and they agree with our results and those in Ref. [25]. We note that the V-type probe transitions in the $Q(1)$ and $R(1)$ overtone lines (indicated in red in Fig. 4) were observed in three different measurement series (performed at 3 and 2 week intervals), with pump locked to the $R(1), Q(1)$, and $P(1)$ lines in the fundamental band. The center frequencies of the $Q(1)$ and $R(1)$ overtone lines from the different measurements agree within their uncertainties, and their standard deviations are 0.1 and $0.47 \mathrm{MHz}$, respectively. This demonstrates the long-term repeatability of the measurements and indicates that the precision of the center frequencies determined using sub-Doppler DR spectroscopy is better than $1 \mathrm{MHz}$, even though the signal-to-noise ratio is at most 10 for the strongest lines. Moreover, the sub-Doppler V-type resonances allow determination of line center positions with the same accuracy even in the case of overlapping Dopplerbroadened lines [e.g., the $P\left(3, F_{2}\right)$ triplet, see Ref. [26] ], which is not possible with Doppler-limited spectroscopy.

The ladder-type probe transitions were found in the spectra using a numerical procedure described in Ref. [26]. Five such transitions were found when the pump was locked to the $R(0)$ transition in the $\nu_{3}$ band (see Fig. 3), and three of them are shown in Fig. 5 for perpendicular (black) and parallel (orange) pump-probe polarizations. The center frequency, peak value, and width of each transition were found by fitting a Lorentzian function to the data, together with a second-order polynomial to model the weak Doppler-broadened background caused by elastic

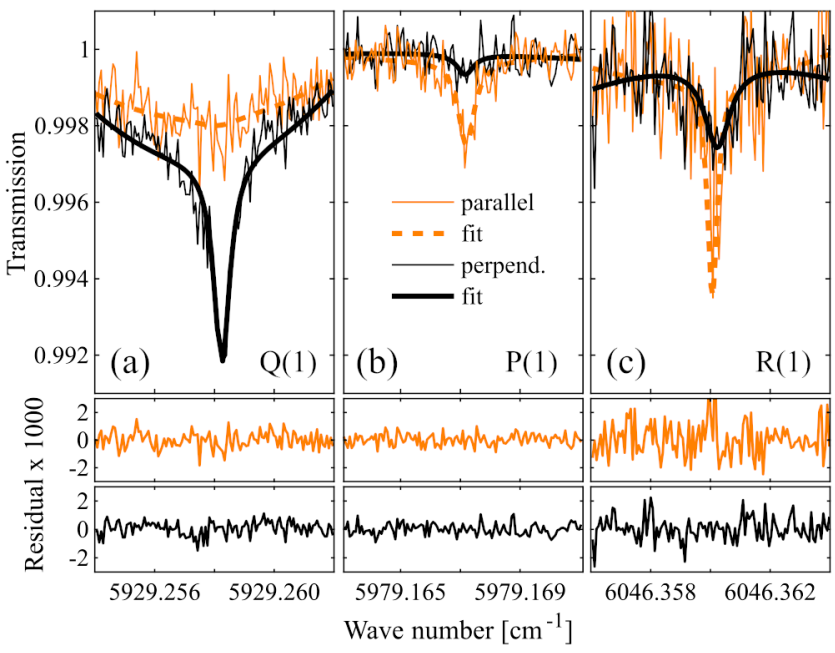

FIG. 5. The (a) $Q(1)$, (b) $P(1)$, and (c) $R(1)$ ladder-type probe transitions observed in the spectrum when the pump is locked to the fundamental $R(0)$ line, recorded with parallel (orange) and perpendicular (black) relative pump-probe polarizations. The thin curves show the data, while the thick curves show the fits (dashed orange for parallel polarization and solid black for perpendicular polarization), and the residuals are shown in the lower panels. collisions. The fits are shown by the thicker curves in Fig. 5 (solid black for perpendicular polarization, and dashed orange for parallel polarization), and the fitting routine and results for all DR transitions are described and summarized in Ref. [26]. The uncertainty of the transition frequencies, calculated as a combination of the fit uncertainty and the stability of the pump, ranges from 1.36 to $3 \mathrm{MHz}$, with a mean of $1.7 \mathrm{MHz}$.

The line assignment indicated in Fig. 5 is based on the ratios of probe transition intensities recorded with parallel and perpendicular pump-probe polarizations. The expected ratios for the pump locked to the $R(0)$ transition, calculated from the ratios of squared transition matrix elements summed over $M$ quantum numbers, are zero for a $Q(1)$ line, infinity for a $P(1)$ line, and 1.33 for an $R(1)$ line [31], in good agreement with observations. The assignment is confirmed by comparison with theoretical predictions from the TheoReTS database [7], which contains rotationally resolved hot methane line lists obtained from ab initio calculations (with empirical corrections to band centers applied when available) [8]. The differences between the center frequencies of the ladder-type probe transitions and predictions from the TheoReTS database are shown in Fig. 6. The estimated accuracy of the predictions is $1 \mathrm{~cm}^{-1}$ and - remarkably - all detected lines are within this range from predictions. We note that the frequency difference between predicted lines sharing the lower level is a few tens of $\mathrm{cm}^{-1}$, allowing unambiguous assignment. In total, 36 ladder-type probe transitions have been identified and assigned. The Ref. [26] summarizes the experimental transition wave numbers, final state values, and widths of all detected lines. The final states are compared to those reached in the work of de Martino et al [20-22] and the transition frequencies and intensities are compared to predictions from the TheoReTS and ExoMol line lists.

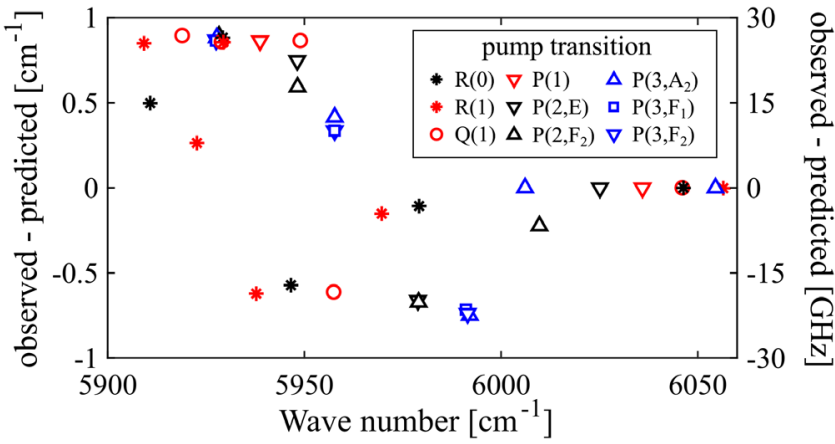

FIG. 6. The difference between center frequencies of the subDoppler ladder-type probe transitions (detected with the pump locked to nine different fundamental transitions, as marked in the legend) and the center frequencies predicted in the TheoReTS database. The left axis is in wave numbers, while the right axis is in gigahertz. The experimental error bars, which are negligible on this scale, are not shown 
In conclusion, we used a high-power continuous wave laser as a pump and a frequency comb as a probe to detect multiple sub-Doppler optical-optical double-resonance transitions in methane. The V-type probe transition frequencies agree to within $0.35 \mathrm{MHz}$ with previous high-precision measurements of the $2 \nu_{3}$ band. The ladder-type probe transitions reaching states in the $3 \nu_{3}$ band region have not been previously observed. We assign them with the help of the TheoReTS line list, and the assignment is confirmed for lines measured with the pump locked to the $R(0)$ line by the ratios of probe transition intensities measured with parallel and perpendicular relative pumpprobe polarization. The center frequencies agree within the estimated accuracy of the theoretical predictions.

The frequency accuracy is currently limited mainly by the stability of the pump frequency $(1.33 \mathrm{MHz})$, which can be improved by optimizing the Lamb-dip lock or replacing it with a direct lock to the comb. The signal-to-noise ratio of the strongest ladder-type probe transitions is currently limited to around ten by the short interaction length. The absorption sensitivity can be increased by implementing an enhancement cavity for the comb probe, as was recently demonstrated in continuous wave optical-optical DR spectroscopy [32]. These improvements will allow detection of a larger number of weaker probe transitions with higher frequency accuracy and better precision on the line shape, thus confirming the assignment for higher $J$ states. The assigned levels in the $9000 \mathrm{~cm}^{-1}$ energy region can serve as stepping stones to access even higher levels in the $15000 \mathrm{~cm}^{-1}$ range using a $1 \mu \mathrm{m}$ pump.

Double-resonance spectroscopy using two monochromatic lasers is a well-established method, but the frequency range that can be probed with it is restricted by the limited tunability of the narrow-linewidth lasers. Using a frequency comb probe, double-resonance transitions can be detected with tens of thousands of narrow comb teeth simultaneously. This technique has been recently employed for measurement of highly excited transitions in $\mathrm{Rb}[33,34]$ over $0.2 \mathrm{THz}$ of bandwidth using the dual comb approach. Here we apply it for the first time to a molecular species, achieving $6 \mathrm{THz}$ of probe bandwidth. The technique, with different configurations of $\mathrm{cw}$ pump and comb probe, is broadly applicable to the assignment of spectra of many molecules, for which spectral perturbations and/or congestion prevent assignment by traditional methods alone. This method allows for accurate assignment of many highly excited molecular energy levels and verification of theoretical predictions of spectral transition frequencies and intensities. Such verification is needed to assess the accuracy of predicted high-temperature spectra for modeling the high-temperature environments of exoplanets. This is particularly relevant for the planned NASA $[35,36]$ and ESA missions [37], which will investigate the temperature structure and chemical composition of the exoplanetary atmospheres using infrared spectroscopy.
The authors thank Jin Guo and Chuang Lu for help with setting up the cw-OPO. A.F. acknowledges the Swedish Research Council (2016-03593) and the Knut and Alice Wallenberg Foundation (KAW 2015.0159). G. S. acknowledges the Foundation for Polish Science (POIR.04.04.00-00434D/17-00). O. A. acknowledges the Swedish Research Council (2015-04374) and the Kempe Foundations (JCK 1317.1).

*aleksandra.foltynowicz@umu.se †kl6c@virginia.edu

[1] M. R. Swain, G. Vasisht, and G. Tinetti, Nature (London) 452, 329 (2008).

[2] M. R. Swain et al., Nature (London) 463, 637 (2010).

[3] A. P. Showman, Nature (London) 452, 296 (2008).

[4] G. Guilluy, A. Sozzetti, M. Brogi, A. S. Bonomo, P. Giacobbe, R. Claudi, and S. Benatti, Astron. Astrophys. 625, A107 (2019).

[5] S. N. Yurchenko and J. Tennyson, Mon. Not. R. Astr. Soc. 440, 1649 (2014).

[6] S. N. Yurchenko, J. Tennyson, J. Bailey, M. D. J. Hollis, and G. Tinetti, Proc. Natl. Acad. Sci. U.S.A. 111, 9379 (2014).

[7] M. Rey, A. V. Nikitin, Y. L. Babikov, and V. G. Tyuterev, J. Mol. Spectrosc. 327, 138 (2016).

[8] M. Rey, A. V. Nikitin, and V. G. Tyuterev, Astrophys. J. 847, 105 (2017).

[9] G. J. Scherer, K. K. Lehmann, and W. Klemperer, J. Chem. Phys. 81, 5319 (1984).

[10] B. Amyay and V. Boudon, J. Quant. Spectrosc. Radiat. Transfer 219, 85 (2018).

[11] A. V. Nikitin, A. E. Protasevich, M. Rey, and V. G. Tyuterev, J. Chem. Phys. 149, 159901 (2018).

[12] M. Ghysels, S. Vasilchenko, D. Mondelain, S. Beguier, S. Kassi, and A. Campargue, J. Quant. Spectrosc. Radiat. Transfer 215, 59 (2018).

[13] A. Wong, P. F. Bernath, M. Rey, A. V. Nikitin, and V. G. Tyuterev, Astrophys. J. Suppl. Ser. 240, 4 (2019).

[14] A. V. Nikitin, A. E. Protasevich, M. Rey, V. I. Serdyukov, L. N. Sinitsa, A. Lugovskoy, and V. I. G. Tyuterev, J. Quant. Spectrosc. Radiat. Transfer 239, 106646 (2019).

[15] R. J. Hargreaves, I. E. Gordon, M. Rey, A. V. Nikitin, V. G. Tyuterev, R. V. Kochanov, and L. S. Rothman, Astrophys. J. Suppl. Ser. 247, 55 (2020).

[16] W. Demtröder, Laser Spectroscopy, 4th ed. (Springer, New York, 2008).

[17] K. K. Lehmann and S. L. Coy, Phys. Chem. Chem. Phys. 92, 306 (1988).

[18] M. S. Feld and A. Javan, Phys. Rev. 177, 540 (1969).

[19] V. S. Petrovic and R. W. Field, J. Chem. Phys. 128, 014301 (2008).

[20] A. de Martino, R. Frey, and F. Pradere, Chem. Phys. Lett. 111, 113 (1984).

[21] A. de Martino, R. Frey, and F. Pradere, Mol. Phys. 55, 731 (1985).

[22] M. de Chevalier and A. de Martino, Chem. Phys. Lett. 135, 446 (1987).

[23] S. Okubo, H. Inaba, S. Okuda, and H. Sasada, Phys. Rev. A 103, 022809 (2021). 
[24] C. Ishibashi, M. Kourogi, K. Imai, B. Widiyatmoko, A. Onae, and H. Sasada, Opt. Commun. 161, 223 (1999).

[25] A. M. Zolot, F. R. Giorgetta, E. Baumann, W. C. Swann, I. Coddington, and N. R. Newbury, J. Quant. Spectrosc. Radiat. Transfer 118, 26 (2013).

[26] A. Foltynowicz et al., companion paper, Phys. Rev. A 103, 022810 (2021).

[27] G. Sobon, T. Martynkien, D. Tomaszewska, K. Tarnowski, P. Mergo, and J. Sotor, Photon. Res. 6, 368 (2018).

[28] A. Foltynowicz, T. Ban, P. Maslowski, F. Adler, and J. Ye, Phys. Rev. Lett. 107, 233002 (2011).

[29] P. Maslowski et al., Phys. Rev. A 93, 021802(R) (2016).

[30] L. Rutkowski, P. Maslowski, A. C. Johansson, A. Khodabakhsh, and A. Foltynowicz, J. Quant. Spectrosc. Radiat. Transfer 204, 63 (2018).
[31] N. Skribanowitz, M. S. Feld, and M. J. Kelly, Phys. Rev. A 6, 2302 (1972).

[32] C. L. Hu, V. I. Perevalov, C. F. Cheng, T. P. Hua, A. W. Liu, Y. R. Sun, Y. Tan, J. Wang, and S. M. Hu, J. Phys. Chem. Lett. 11, 7843 (2020).

[33] A. Nishiyama, S. Yoshida, Y. Nakajima, H. Sasada, K. Nakagawa, A. Onae, and K. Minoshima, Opt. Express 24, 25894 (2016).

[34] A. Nishiyama, Y. Nakajima, K. Nakagawa, A. Onae, H. Sasada, and K. Minoshima, Opt. Express 27, 37003 (2019).

[35] J. Kalirai, Contemp. Phys. 59, 251 (2018).

[36] https://jwst.nasa.gov/index.html.

[37] https://arielmission.space/. 